\title{
A Small World Overlay Network for Resource Discovery
}

\author{
Koen Vanthournout, Geert Deconinck, and Ronnie Belmans \\ Katholieke Universiteit Leuven, \\ Department Electrical Engineering (ESAT), Belgium \\ Koen.Vanthournout@esat.kuleuven.ac.be \\ http://www.esat.kuleuven.ac.be
}

\begin{abstract}
Interest is rising in genuinely distributed ('peer-to-peer') resource discovery systems, which all provide mechanisms for self-organization of their resource providers and requesters into an overlay network. This paper proposes such a system that uses XML description files to construct overlay networks that exhibit small world properties (i.e., a small diameter and a regular structure) and in which similar resources are grouped together. Resource users can then be linked directly to the regions with the resources of their interest, which allows exploitation of group and time locality. Simulations results validate these concepts.
\end{abstract}

\section{Introduction}

Resource discovery (RD) is an indispensable enabling technology for networked distributed systems. As such, a multitude of existing technologies provide the required functionalities and these can be divided into roughly two clusters: thirdparty systems and genuinely distributed systems. For the first type, a separate entity, independent from the resource providers and requesters, gathers references to resources and solves resource queries (e.g., LDAP [6], Matchmaking [8], etc.). Genuinely distributed systems, on the other hand, are embedded in the users (both resource providers and requesters) of the system (e.g., Gnutella [5], Freenet [4], CAN [9], etc.).

The field of third party RD systems is well established, in contrast to the fairly new genuinely distributed approach. The latter has distinct advantages, though:

- There is no dependency on a central coordinating authority.

- Every participant makes some resources available and, consequently, the available resources grow with the number of participants, which allows for scalable systems.

- The larger the number of participants, the larger the inherent redundancy in the system, which can be exploited to achieve increased fault-resilience.

All these genuinely distributed resource discovery systems or, so called by popular consensus, peer-to-peer systems compose of two main components: an overlay network and a query resolving technique. A closer look at these components of 
the peer-to-peer RD systems reveals a number of short-comings. Gnutella [5], by far the most spread system, deploys a random overlay structure and uses flooding to propagate queries, both being inefficient. Freenet [4] nodes are (initially) also gathered in a random structure, but the use of local caches of files with similar keys and single query forwarding to the neighbor node with the closest matching keys, allows convergence of a Freenet network to a more efficient structure. But it can only be used for files and not for other (non-copyable) resources, e.g., processing servers. A third type of systems (CAN [9, 12], Chord [11], Pastry [10], Tapestry [17] and P-Grid [2]) uses regular overlay network structures. A unique identifier is assigned to all nodes and this is then used to position the nodes on a predictable position in the network, whose structure reflects the identifier space. An ID is computed for each resource and a reference to the position of those resources is then stored at the node with the closest ID. The result is a search-optimized overlay network. But one can predict difficulties with variable resources: if the description of a resource changes, so does its identifier and, potentially, the position of its reference. This is a symptom of the fact that there is no or little correlation between the ID's and the nature and type of the resources. Functionality is not reflected in the structure of the overlay networks and, consequently, neither group locality, nor time locality [7] are exploited:

- group locality: Requesters can be divided into groups that tend to search for the same types of resources.

- time locality: The same entity may request the same or comparable resources several times within short time intervals.

Mentionable is the work presented in [15], which proposes methods to construct an overlay network that links nodes that are interested in similar documents. Their usefulness is limited, though, to file-sharing systems: it assumes that nodes interested in a certain resource (in this case, file) also provide similar resources.

Hence, there is a need for a distributed RD system that supports resources with variable properties, uses attribute naming ${ }^{1}$ and where the nodes self-organize into a regular structure that reflects the nature of the resources [14].

The system that is proposed in this paper fills this gap. It uses a metric based on the differences between descriptions in XML of the resources, to construct an overlay network in which nodes with a comparable functionality are grouped together and in which resource users are close to the resources for which they announced an interest. The next section describes the construction of this overlay network, followed by the query forwarding strategy. Simulation results are presented at the end to illustrate the usefulness of this approach.

\section{The Overlay Network}

\subsection{Distance Between Functionalities}

A desirable property for RD overlay networks is regularity, because a regular, predictable structure will ease and optimize query forwarding. But to allow nodes

\footnotetext{
${ }^{1}$ Attribute based queries in, e.g., XML, offer a more powerful search mechanism than hash-based ID's.
} 
to self-organize into such a regular network, some distance-value is needed: if new nodes can calculate the distance from the position they should be in the regular structure to any other node they encounter in that network, they can hop through the network, from node to node, until they arrive at their proper position, where they can form links to their surrounding neighbors. The same goes for resource queries: if a node can calculate the distance from its position to the place in the network where the query can be answered, then it can select its neighbor closest to that place and forward the query there. This is the strategy deployed by CAN, Chord, Pastry, P-Grid and Tapestry. But, as discussed before, they all deploy a regular identifier space and a unique ID per node that has no semantic correlation to the nature and type of the resources.

The system, here proposed, employs a method to calculate a distance that does reflect this: all nodes contain an XML description file with all its resources, be it files, processing power, printing services, domotica device or any other conceivable resource, and a list of their main interests; the resources they are likely to request. The distance between two nodes and, thus, between their XML description files $\left(\mathcal{D}\left(x m l_{1}, x l_{2}\right)\right.$ is then defined as:

$$
\mathcal{D}\left(x m l_{1}, x m l_{2}\right)=1-\frac{\operatorname{size}\left(\mathcal{N}_{\text {common }}\right)}{\operatorname{size}\left(\mathcal{N}_{\text {common }}\right)+\operatorname{size}\left(\mathcal{N}_{n c, x m l_{1}}\right)+\operatorname{size}\left(\mathcal{N}_{n c, x m l_{2}}\right)}
$$

with $\mathcal{N}_{\text {common }}$ the set of common XML nodes, where a XML node is defined as common iff the node and all its ancestors exist in both files. Text and values can only be contained by leaf nodes and count as an separate node for the purpose of the distance metric. Attributes are ignored. *, which yields an automatic match, is allowed for text fields in interest and query XML (see Sections 2.4 and 3). $\mathcal{N}_{n c, x m l_{n}}$ is the set of non-matching nodes for XML file $\mathrm{n}$.

An example description file of a server offering processing cycles:

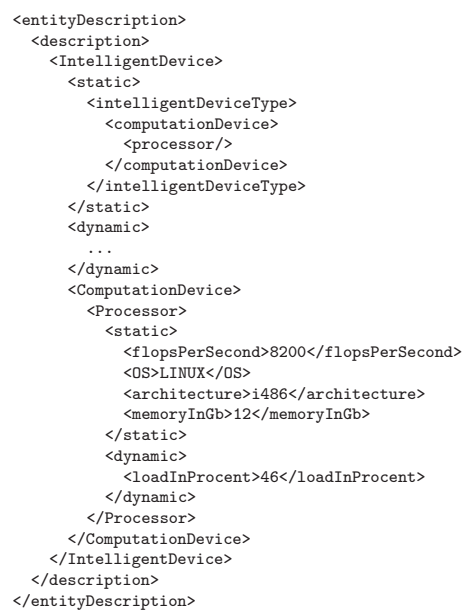

This metric is at the same time the novelty, the strength, but also the weakness of this RD system: it guarantees the construction of a semantic overlay network, but ill-constructed XML will deteriorate that very construction. As such, semantic 
gossiping [1], which is designed to overcome this type of problems, would be very useful. Secondly, the above described metric is simple and lean and serves the purpose of concept-proof well, though a real-life system will require a more robust metric with more stringent syntax rules and an expanded set of masks $(<,>, !=$, etc. $)$. But that is beyond this paper's scope.

\subsection{Companions}

The companions of a node are the nodes that offer the most similar resources: the distance metric, applied on the resource descriptions only, yields the smallest results. Every node looks for a fixed number of companions (this number defines the dimensionality of the overlay network) and these companions indicate the correct position of that node in the network.

When a new node enters the system, the only initialization needed is the address of an active node of the network. To this initial neighbor, it transmits a companion request, which contains the requester's XML description, the highest distance metric of its current neighbors (the threshold), the addresses of its current neighbors and a hop limit. A node that receives such a request checks if it knows any nodes with a lower distance metric result for the requester than the threshold, that are not in the neighbor list attached to the request. If so, it replies their addresses to the requester. If the hoplimit has not been reached, the request is forwarded to all nodes that matched the request, if any. Else a single message is send to the neighbor with the lowest metric result. If a message is received for a second time, it is dropped.

The requester listens to answers for a fixed time, after which it selects its new neighbors from the replies it received. It announces the construction of the new links to those new neighbors and then repeats the whole cycle by retransmitting a companion request. Convergence is reached when it receives no answers any more: its current neighbors are its companions. Because of the link announcements, these companions can update their links to the newcomer. Furthermore, all nodes periodically restart the convergence cycle, as to cope with changes in the network's node composition.

\subsection{Small Worlds and Far Links}

If all nodes would only locate their companions and solely form links to those, the result would be network partitioning and, because of the high regularity, a high network diameter and characteristic path length (see Section 4). This can be avoided by the use of far links: a fraction of the nodes constructs a link to the node that yields the worst result for the distance metric. The impact of this on the network's regularity is minor, but the network's diameter decreases significantly $[3,16]$ : a small world topology emerges, which combines the best of random networks (a low diameter) and regular networks (a predictable structure). And it is an effective prevention against network partitioning. Their construction is equal to the construction of companion links, but with word 'lowest' replaced by 'highest' and vice versa. 


\subsection{Resource Providers and Resource Users}

The above-described links will result in an overlay network that reflects the functionality: nodes are clustered by functionality and, as a result, the answers to queries for comparable resources are to be found in the same region. Next optimization is to link resource requesters to the regions of their interest. Each resource requester constructs a number of links (predetermined) to the nodes whose descriptions match its expressions of interests best. This is realized by using the same convergence method as described in Section 2.2, but the metric now compares the XML expressions of interest of the requester to the description of the resources of the potential recipients of these links.

\subsection{Orphans}

One problem emerges after above links are formed. If a node deviates from the bulk of the nodes it resembles, then it might become an orphan, i.e., it does link to the nodes of its kind, but it is too different for other nodes to refer to it (if there happens to be no far link or resource user link to it) and thus the node becomes unreachable. The solution to this is to have each node also link to the last nodes that announced their link and to which this node has no link already (announcements are periodically repeated). Experiments show that one such anti-orphan link per node eliminates the existence of orphans, though a node can still be temporary unreachable if a new node, in the process of converging to its proper spot, traverses the network and 'steals' anti-orphan links on its way.

\section{Resource Queries}

A query consists of the address of the requester, the XML file that contains the query and a hoplimit. A query XML file contains the description of a resource, possibly with masks. Only fully matching resources (distance metric is zero) yield a match.

When a node receives a query, then it checks if itself or any of its neighbors matches the XML file. If so, a query answer is send to the requester. If not and if the hoplimit is not yet reached, the query is forwarded to the closest matching neighbor.

\section{Results}

In order to optimize and evaluate the system, it was simulated on top of an in-house developed network simulator, for various numbers of nodes. Nodes are connected in random sequential order to the network with random time slots between the additions. They are initially connected to a random active node. After all nodes have been added, the simulation continues for sufficient time to allow all nodes to converge. Then following metrics are calculated: 

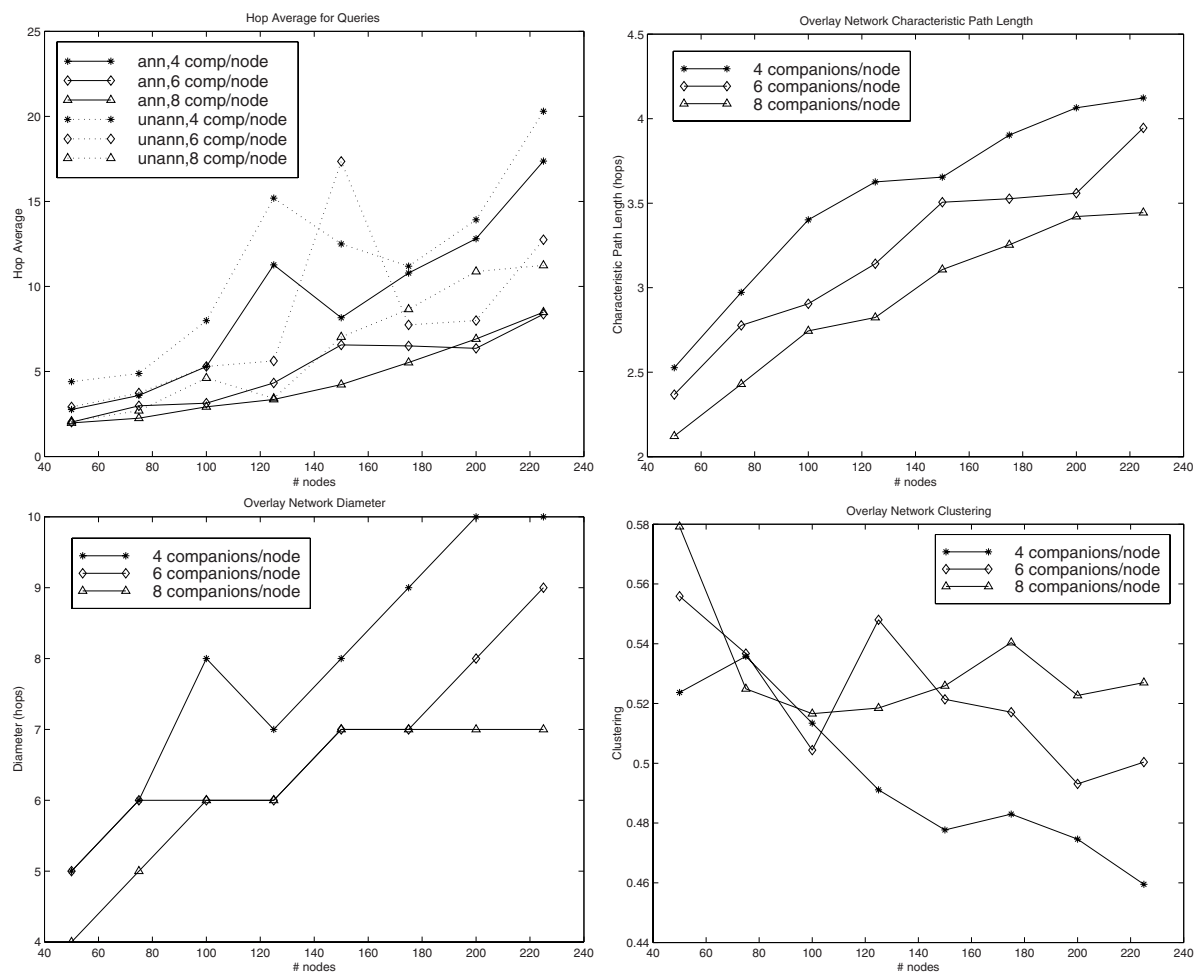

Fig. 1. The average number of hops per queries, characteristic path length, diameter and clustering for overlay networks with different numbers of companions per node (ann: the node announced an interest for the query's class of resources, unann: no interest was announced). $50 \%$ of the nodes form a far link and all nodes construct one anti-orphan link.

- Clustering $(C)$ [16,3]: A node $i$ has $k_{i}$ edges to $k_{i}$ other nodes. If this node and its neighbors would be part of a clique, there would be $k_{i}\left(k_{i}-1\right)$ edges between them (for a directed graph). $E_{i}$ is the number of edges that actually exist between these $k_{i}$ nodes. The clustering coefficient of node $i$ is then: $C_{i}=E_{i} /\left(k_{i}\left(k_{i}-1\right)\right)$. The clustering coefficient of the whole network is the average of all individual $C_{i}$ 's. It is an indication of how regular the network is.

- Characteristic path length $(L)$ [16]: The characteristic path length $L$ of a graph $G$ is the average of the number of edges in the shortest path between all pairs of vertices. $L$ is lowest for random networks and high for perfect regular structures. It is a property of small world networks to have a high $C$ and at the same time a low $L$, which means that they possess the positive properties of both regular and random networks.

- Diameter $(D)$ : The diameter of a graph is equal to the longest shortest path in the graph. 
- Hop average for queries: Every network is tested with a batch of queries with a size proportional to the number of nodes in the network. These are divided into two categories: queries from nodes for resources for which they announced an interest and queries from nodes for resources for which they did not. The average number of hops a query travels before an answer is found, evaluates the network's efficiency.

Figure 3 gives these metrics for networks with 4, 6 and 8 companions per node. Less than four companions per node results in networks with deficient properties for larger numbers of nodes. The results show that indeed small world networks are constructed: the clustering coefficient stays between 0.46 and 0.58 , while the characteristic path length stays as low as 4.12 for 4 companion per node networks of size 225 and 3.44 for 8 companion per node networks of the same size.

The average number of hops a query travels illustrates the usefulness of announcing interests. Only three clusters of resources were used for the simulations, but already a clear profit is made, as can be seen in the larger average number of hops needed for queries to unannounced resource than for queries to announced resources. As the diversity of the resources rise, more distinct results can be expected.

\section{Conclusions and Future Work}

The use of XML description files and the differences between those, allows for the construction of small world networks in which nodes with similar files are clustered together. This can be used for distributed resource discovery systems that exploit group and time locality: resource users can be linked directly to regions where the resources of their interest are located. Simulations confirmed the validity of this concept. Further simulations with larger numbers of nodes and investigation of the convergence algorithms, used for the positioning of nodes and for query forwarding, will provide additional information on scalability. The effects of node and message failures have been studied and are presented in [13].

\section{Acknowledgements}

This work is partially supported by the K.U.Leuven Research Council (GOA/ 2001/04) and the Fund for Scientific Research - Flanders through FWO Krediet aan Navorsers 1.5.148.02.

\section{References}

1. K. Aberer, P. Cudré-Mauroux, and M. Hauswirth. A framework for semantic gossiping. SIGMOD Rec., 31(4):48-53, 2002.

2. K. Aberer, A. Datta, and M. Hauswirth. Efficient, self-contained handling of identity in peer-to-peer systems. IEEE Transactions on Knowledge and Data Engineering, 16(7):858-869, Jul 2004. 
3. Réka Albert and Albert-Lásló Barabási. Statistical mechanics of complex networks. Reviews of Modern Physics, 74:47-97, Jan 2002.

4. I. Clarke, O. Sandberg, et al. Freenet: A distributed anonymous information storage and retrieval system. LNCS, 2009:46-66, 2001.

5. Gnutella. The gnutella protocol specification. http://rfc-gnutella.sourceforge.net.

6. T. Howes and M. Smith. Rfc 1823: The ldap application program interface. http://www.faqs.org/rfcs/rfc1823.html, Aug 1995.

7. A. Iamnitchi, M. Ripeanu, and I. Foster. Locating data in (small-world?) peerto-peer scientific collaborations. In 1st Int'l Workshop on Peer-to-Peer Systems (IPTPS'02), Cambridge, MA, Mar 2002.

8. Rajesh Raman, Miron Livny, and Marvin Solomon. Matchmaking: Distributed resource management for high throughput computing. In 7th IEEE Int'l Symposium on High-Performance Distributed Computing, 1998.

9. S. Ratnasamy, P. Francis, et al. A scalable content addressable network. In Proc. of ACM SIGCOMM, pages 161-172, San Diego, USA, Aug 2001.

10. A. Rowstron and P. Druschel. Pastry: Scalable, decentralized object location, and routing for large-scale peer-to-peer systems. In IFIP/ACM Int'l Conference on Distributed Systems Platforms, pages 329-350, Heidelberg, Germany, 2001.

11. Ion Stoica, Robert Morris, David Liben-Nowell, et al. Chord: A scalable peerto-peer lookup protocol for internet applications. IEEE/ACM Transactions on Networking, 11(1):17-32, Feb 2003.

12. C. Tang, Z. Xu, and S. Dwarkadas. Peer-to-peer information retrieval using selforganizing semantic overlay networks. In Proc. of the 2003 conference on Applications, Technologies, Architectures, and Protocols for Computer Communications, pages 175-186. ACM Press, 2003.

13. Koen Vanthournout, Geert Deconinck, and Ronnie Belmans. Building dependable peer-to-peer systems. In Twin Workshops on Architecting Dependable Systems (WADS 2004), Florence, Italy, Jun 2004. Accepted for publication and presentation.

14. Koen Vanthournout, Geert Deconinck, and Ronnie Belmans. A taxonomy for resource discovery. Lecture Notes in Computer Science, 2981:78-91, Mar 2004.

15. S. Voulgaris, A.-M. Kermarrec, L. Massoulie, and OTHERS. Exploiting semantic proximity in peer-to-peer content searching. In Proc. 10th IEEE Int'l Workshop on Future Trends in Distributed Computing Systems (FTDCS), May 2004.

16. Duncan J. Watts and Steven H. Strogatz. Collective dynamics of 'small-world' networks. Nature, 393:440-442, 1998.

17. Ben Y. Zhao, Ling Huang, Jeremy Stribling, et al. Tapestry: A resilient global-scale overlay for service deployment. IEEE Journal on Selected Areas in Communications, 22(1):41-53, Jan 2004. 